\title{
Research on Optimization Design of WNN modeling Based on Modified AFSA
}

\author{
Wang Pingan ${ }^{1, a}$, Gan Xusheng ${ }^{2, b}$, Li Huaping ${ }^{3, c}$ \\ ${ }^{1 .}$ XiJing College, Xi'an, Shaanxi, 710123, China; \\ 2. Air Traffic Control and Navigation College, Air Force Engineering University, Xi'an, Shaanxi, 710051, \\ China; \\ 3. PLA, Air Force, Xi' an Flight Academy, Xi'an, Shaanxi, 710306 \\ a32433122@qq.com; bganxusheng123@163.com; ㄷww2121@sohu.com
}

Keywords: RBF Neural Network, Artificial Fish Swarm Algorithm, Stock Price Trend, Prediction.

\begin{abstract}
For the parameter initialization and network structure determination of Wavelet Neural Network (WNN) modeling, an optimization design method based on modified Artificial Fish Swarm Algorithm (AFSA) is proposed. Firstly, the effect of initial parameters and network structure on the performance of WNN model is discussed, and then an AFSA with different fish foraging behavior is applied to determine the initial parameters and the number of hidden nodes required in modeling. The experiment shows that, the proposed method can completely solve the optimization design problem in WNN modeling.
\end{abstract}

\section{Introduction}

The convergence rate and generalization ability are two most important performance indices of Wavelet Neural Network (WNN) model. Research shows that the convergence speed of WNN mainly depends on the parameter initialization, the initial value selected is reasonable, the convergence speed is fast; otherwise, the convergence is slow, the algorithm is easy to fall into the local minimum, and even oscillating and not convergence. And its generalization ability depends mainly on the network topology structure (for the typical three-layers WNN, it mainly refers to the number of hidden nodes). The number of hidden nodes selected is too less, the algorithm cannot fully learn the sample and cannot achieve the specified accuracy; If the number of hidden nodes is too large, The algorithm is prone to generalization performance degradation and over-fitting phenomenon. In the use of WNN model to solve specific practical problems, the above parameters initialization and network structure problems can be collectively referred to as the problem of WNN optimization design.

Based on this, the effect of parameter initialization and network structure on the performance of WNN is discussed, and a optimization design method of WNN based on improved Artificial Fish Swarm Algorithm (AFSA) is proposed to obtain the reasonable initial parameters and the optimal number of hidden nodes at the same time. The feasibility and effectiveness of the method are verified by simulation examples.

\section{Wavelet Neural Network}

WNN is the product of the combination of neural network idea and wavelet analysis theory. It has also been proved that WNN has the better approximation ability than traditional neural network and overcomes many disadvantages of neural network.

Assume that $\psi$ is the wavelet basis that satisfied the admissibility condition and $f(x) \in L^{2}(R)$, then for the multiple inputs and multiple outputs, the output of network is 


$$
y_{k}(x)=\sum_{j=1}^{J} v_{k j} \psi_{j}\left(\frac{\sum_{i=1}^{I} w_{j i} x_{i}-b_{j}}{a_{j}}\right)
$$

where $a_{j}$ is the scaling factor, $b_{j}$ is the translation factor.

For a WNN structure with three-layers as shown in Fig. 1, $x_{i}$ is the input nodes, $y_{k}$ is the output nodes, $v_{k j}$ is the connection weight between hidden nodes and output nodes, $w_{j i}$ is the connection weight between input nodes and hidden nodes, $I, J, K$ are the number of input nodes, hidden nodes, and output nodes respectively. In nature, the training of WNN can be thought of as the optimal estimate process of $w_{j i}, v_{k j}, a_{j}, b_{j}(i=1,2, \cdots, I ; j=1,2, \cdots, J ; k=1,2, \cdots, K)$, and the different optimization algorithm used can come into being different WNN learning algorithm, usually, the gradient descend algorithm is used to optimize the parameters of WNN.

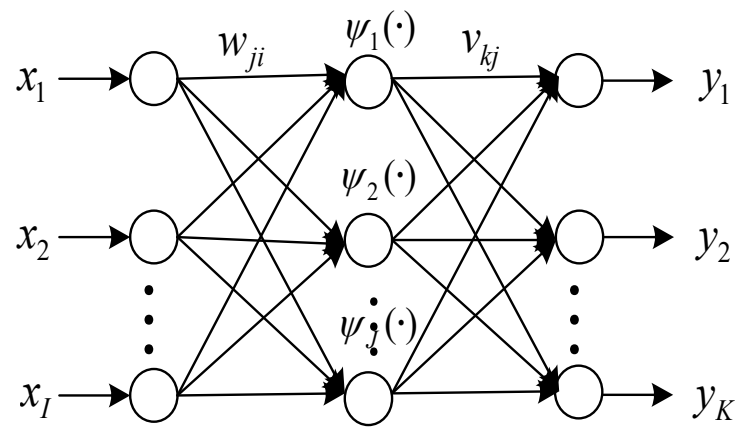

Fig. 1. Structure of WNN

\section{Parameters Initialization and Network Structure of WNN}

WNN has many advantages over neural networks. However, because WNN theory is not yet mature, so far there is no set of perfect theory to guide its optimization design process. The optimization design of $\mathrm{WNN}$ is mainly focused on parameter initialization and network structure determination.

\subsection{Effect of Parameter Initialization}

The theoretical analysis and numerical experiments show that, the initial weight setting of neural network has great influence on the training effect. The initial weight set well, can speed up the convergence rate; otherwise, the number of learning will be greatly increased, and the algorithm even do not converge. Because the analytic, continuous, differentiable and explicit expression between initial weights and network performance cannot be obtained, the initial weights are randomly generated, which is usually not guaranteed to obtain an excellent initial weights, or can only happen by chance. In more cases, the initial weight obtained by such way is easy to make the network error into a hovering, the number of learning substantially increases, and it is not even convergence. As an important branch of neural network, WNN also faces the same problem. The initial value setting of connection weights, translation factors and scaling factors have a great impact on network convergence rate. If set incorrectly, it may lead to slow convergence, or even no convergence. At present, there are few literatures to study the initial value of WNN parameters. In some literatures, two empirical methods are proposed to improve the initial value of the parameters [1][2]. However, it is obviously not guaranteed for ideal initial value of parameters.

\subsection{Effect of Network Structure}

For a three-layer WNN structure with one input layer, one hidden layer, and one output layer, determining the network structure refers to determining the number of hidden nodes of WNN. The number of hidden nodes is usually related to the requirements of the problem, the number of input and output nodes. If the number of hidden nodes is too large, the training time will be too long, especially in the later stage of training, the error in learning samples may affect the training 
convergence direction, resulting in the deviation for global optimal point, which may reduce the generalization ability, and even lead to over-fitting; if the number of hidden nodes is too less, it may not be able to fully learn the sample, resulting in under-fitting, or even no training. Therefore, determining the number of hidden nodes is a more complex problem. Presently, except for the incremental descending method, there are some methods based on the characteristics of WNN [3]. Although some results are achieved, these methods are not ideal in the application of the more complex problems. Therefore, how to determine the optimal number of hidden nodes for optimal network structure is still the key to wide application of WNN.

\section{Optimization Design Idea of WNN}

AFSA is a new intelligent evolution algorithm. The algorithm is simple, easy to implement, fast and adaptable. The whole calculation process only uses the objective function value and no gradient information. However, similar to genetic algorithm, particle swarm algorithm and so on, AFSA also has the disadvantage that the convergence is obviously slowed near the global optimal solution.

Based on the influence of parameter initialization and hidden nodes number on the performance of WNN, an optimization design method of WNN based on modified AFSA is proposed. Firstly, for the shortcomings of the standard AFSA, the necessary improvement is made. Then, the improved algorithm is used to perform the rough search in WNN parameters and structure space, when searching near the global optimal point, the solution value at this time can be regarded as the initial value and the number of hidden nodes of WNN optimization design. Finally, the gradient descent method (conjugate gradient method, Newton algorithm, quasi-Newton algorithm, etc.) is applied to perform the fine search for the global optimal solution of the network. The design method combines AFSA and the gradient descent method to determine the reasonable initial value of WNN parameters, and can automatically determine the number of hidden nodes.

\section{Modified FSA}

AFSA was first proposed by X. L. Li in 2001, which is an intelligent optimization algorithm for simulating the foraging behavior of fish. The basic principle is to learn from the fish foraging, clustering and rear-end behavior, from the start of constituting the bottom behavior of single artificial fish, the global optimal value can be obtained through local optimization of each individual in fish swarm [4]. Once the algorithm appears, it attracts attention and puts forward some modified AFSA, and it is also widely used in the fields of pattern recognition and parameter optimization.

In main 3 behaviors of artificial fish, the foraging behavior lays the foundation of the algorithm convergence. The clustering behavior enhances the stability and globality of the algorithm convergence. The rear-end behavior improves the convergence and globality of the algorithm convergence. The behavior evaluation also provides a guarantee for speed and stability of the algorithm convergence. In general, the algorithm is still very tolerant of the value range of parameters, and the initial value of the algorithm is basically no requirement.

The specific steps of AFSA are as follows:

1. Initialization of fish swarm. Randomly generate the artificial fish individual in the feasible field of control variables, forming the initial fish swarm.

2. Set the bulletin board. The bulletin board used to record the optimal value the corresponding individual state found by artificial fish swarm.

3. Evaluate the food concentration at the position of each individual in fish swarm.

4. Each individual simulates and performs rear-end behavior and clustering behavior respectively. The behavior with larger food concentration after action is selected as the actual implementation, the foraging behavior is regarded as the default behavior.

5. Update the bulletin board. Each individual check their own food concentration and the food concentration value of bulletin board after every action. If it is better than the bulletin board, then replace it. 
6. Judge whether to stop the iteration. If so, output the result; otherwise, go to step 3.

To improve the performance of AFSA, the foraging behavior can be modified. In the modified algorithm, the artificial fish randomly selects a state $X_{j}$ in its visible domain. If it is found that $X_{j}$ is better than the current state $X_{i}$, it progresses further toward the state $X_{j}$ so that $X_{i}$ achieves a new good state; otherwise, $X_{i}$ continues to randomly reselect the state $X_{j}$ in its visible domain to judge whether to meet the forward conditions. Repeatedly try many times, if you still do not find a better state, then it progresses further toward the best state in current bulletin board. This does not only extend the search range of the algorithm, but also make full use of the favorable information already obtained, thus speeding up the convergence rate of the algorithm.

\section{Algorithm Parameters Setting}

The parameters of AFSA include: fish swarm size, visible domain visual, step size step, crowding factor $\delta$ and number of attempts try-number. It should be noted that inappropriate parameter settings may directly affect the optimization results of algorithm, and even lead to non-convergence. AFSA is a new intelligence swarm optimization algorithm. Because of the wide application of particle swarm algorithm and genetic algorithm, we can learn from the empirical results. However, AFSA is highly dependent on the studied problem, Different optimization problems need to set different parameters, and it has not too much reference. The selection of parameters has a significant effect on the performance of the algorithm.

The impact of these parameters on the convergence characteristics of the algorithm is briefly described as follows:

1. Fish swarm size: In general, the more the number of artificial fish, the greater the ability to jump out of the local extreme, the faster the convergence (from the number of iterations), but the greater the calculation amount for each iteration, therefore, under the premise of satisfying stable convergence, the number of individuals should be reduced as much as possible. Considering the calculation accuracy and calculation speed, the fish swarm size can be generally taken as dozens.

AFSA is an application of swarm intelligence, which is the root cause that the algorithm has some of its own characteristics, the most important of which should be the concept of the swarm, the more the number of artificial fish, the stronger the ability to jump out of the local extreme, the faster the convergence rate, of course, the greater the calculation amount for each iteration, so in the process of the application, under the premise of meeting stable convergence, the number of individuals should be reduced as much as possible.

2. Visible domain visual: The parameter has a great influence on the convergence performance of the algorithm. If it is too small, the foraging behavior and the random swimming of the fish swarm are more prominent. At this time, the optimization process of the algorithm shows a strong randomness, which cannot guarantee the search direction to proceed to the optimal solution. If it is too large, the clustering behavior and the rear-end behavior of the fish swarm are more prominent. At this time, the ability of the algorithm to jump out of the local extremum is weak and easy to fall into the local extremum. Therefore, the visible domain visual must be controlled within a certain range.

3. Step size step: The parameter has great influence on the convergence speed and convergence accuracy of the algorithm. At the early stage of the search, step is too large, the algorithm can get the fast convergence, however, at the later stage of the search, with the clustering of fish to the optimal solution area, the large step size may reduce the local search ability of the algorithm in the optimal solution area, and cannot find the accurately optimal solution. If step is too small, the climbing speed of is very slow.

4. Number of attempts try-number: The parameter is the parameter when the artificial fish individual performs the foraging behavior. - the smaller try-number of forging behavior, the larger the number of random searches of fish swarm, the stronger the ability to jump out of local extremes; the larger try-number of forging behavior, the weaker the ability of the algorithm to overcome local extremes, but the higher the convergence efficiency.

5. Crowding factor $\delta$ : The parameter is used to limit the clustering size of the artificial fish 
swarm, and it is desirable to gather more artificial fish in the neighborhood of the superior state, and in the neighborhood of the suboptimal state it is expected to gather less artificial fish or do not gather artificial fish.

\section{Optimization Design of WNN Based on Modified AFSA}

The steps of optimization design of WNN solved by modified AFSA are as follows:

1. Initialize the fish swarm size $M$, the position of each artificial fish, the visible domain visual, the step size step, factor $\delta$, the number of attempts try-number, the maximum number of iterations number and other parameters.

2. The initial iterations number can be set as num $=0$, and $M$ individuals of artificial fish is randomly generated in the feasible area of the control variable to form the initial fish swarm, that is, generate $M$ groups $w_{j i}, v_{k j}, a_{j}, b_{j}$ and $J$, and except for the variable $J$ as integer, the other variables can be taken as the random number in $[-1,1]$.

3. Calculate the fitness of each artificial fish, and compare with the state in the bulletin board, if better, it is assigned to the bulletin board.

4. Calculate the visible domain visual and the step size step.

5. Each artificial fish updates its position by foraging, clustering, rear-end and random behavior.

6. To judge whether the maximum iterations number number has been reached. If satisfied, the algorithm is terminated, the calculation result is output (that is, $F C$ value of the bulletin board); otherwise, go to step 3.

7. The optimal solution obtained by modified AFSA is decoded as the initial parameter value and the number of hidden nodes of WNN. On this basis, the gradient descend algorithm is used to optimize the network parameters, finishing the training of WNN.

\section{Experiment Simulation}

The function to be approximated is a more complex nonlinear piecewise function

$$
f(x)= \begin{cases}-2.186 x-12.864 & x \in[-10,-2) \\ 4.246 x & x \in[-2,0) \\ 10 e^{-0.05 x-0.5} \sin [(0.03 x+0.7) x] & x \in[0,10]\end{cases}
$$

Suppose that the training set contains 100 points following the uniform distribution in [-10, 10], that is $\left\{\left(x_{1}, y_{1}\right), \cdots,\left(x_{100}, y_{100}\right)\right\}$, the input is $x_{1}, x_{2}, \cdots, x_{100}$, the output is $y_{1}, y_{2}, \cdots, y_{100}$. The input of test set is by 100 points randomly generating in $[-10,10]$, and the test output is the function value of corresponding to the formula (2).

In AFSA, the initial parameters of WNN (connection weights, translation factors and scaling factors) are random numbers in $[-1,1]$, and the number of hidden nodes can be taken as an integer. The fish swarm size $M=40$, the visible domain visual $=1$, the step size step $=0.05$, the crowding factor $\delta=0.168$, the number of attempts try-number $=30$, number $=100$, the number of WNN training by gradient descent method $N=200$, the target error MSE is 0.0001 .

Fig. 2 and Fig. 3 are the function approximation curve and the function approximation error obtained by WNN model, '-' is the function reference curve, and '---' is the network approximation curve. Fig. 4 shows the training convergence curve for WNN.

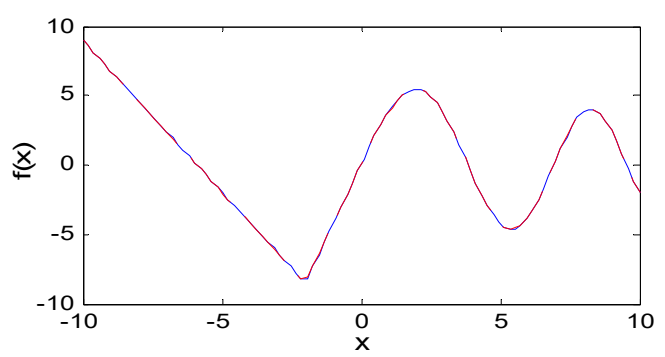


Fig. 2. Function approximation curve

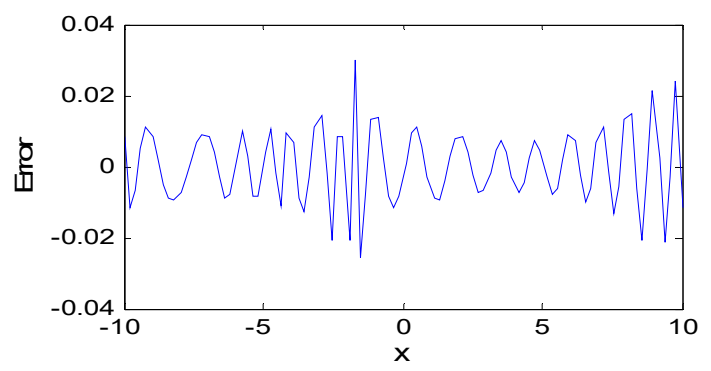

Fig. 3. Function approximation error

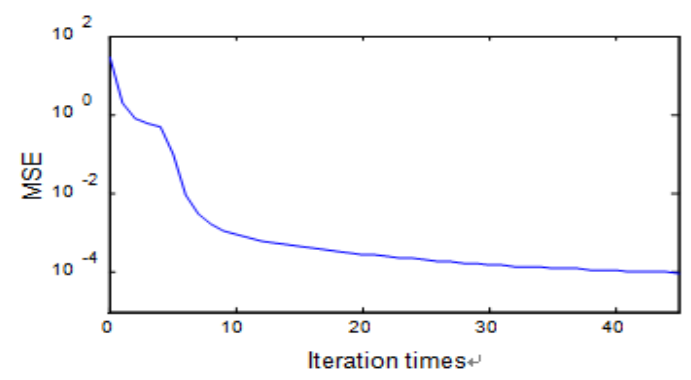

Fig. 4. Training convergence curve

\section{Conclusion}

To solve the problem of optimization design of WNN, firstly, the effect of parameter initialization and network structure on the performance of WNN model is analyzed, then, an optimization design idea based on modified AFSA is proposed and the algorithm parameters setting is discussed, finally, the optimization design method of WNN is validated by the actual example. The result indicates that the modified AFSA can provide the reasonable initial parameters and determine the number of hidden nodes for the modeling of $\mathrm{WNN}$, and it has also proved that the proposed method is effective and feasible.

\section{References}

[1] O. Yacine, D. Gerard, "Initialization by selection for wavelet network training," , vol. 34, No. 1, 2013, pp. 131-143.

[2] X. Z. Zhao, C. H. Zou, T. J. Chen, "A research on the initialization of parameters of wavelet neural networks," Journal of South China University of Technology, vol. 31, No. 2, 2003, pp. 77-79.

[3] M. Han, J. Yin, "New algorithm for node selection in hidden layer of wavelet networks," Journal of System Simulation, vol. 19, No. 17, 2007, pp. 3899- 3902.

[4] X. L. Li, J. X. Qian, "Studies on artificial fish swarm optimization algorithm based on decomposition and coordination techniques," Journal of Circuits and System, vol. 8, No. 1, 2003, pp. 1-6. 